\title{
Possible functions of P21 CDKN1A within the germ cells of the mouse seminiferous epithelium
}

\author{
Ana Pedro ${ }^{1}$ \\ University of Hull, United Kingdom \\ Roma Pharmacy Services, Ltd/ Cymru Digital Health, United Kingdom
}

\begin{abstract}
The cyclin dependent kinase inhibitor P21 CDKN1A has been shown to be upregulated during differentiation and after DNA damage in somatic cells. We examined the expression of P21 CDKN1A and of $\gamma$-H2AX during the differentiation of germ cells in normal mouse testis. P21 CDKN1A was normally expressed in in a variable mode in different cell types (spermatogonia, spermatocytes, spermatids, Sertoli cell) located since the basal till the lumen of the seminiferous tubules. Moreover, in agreement with $\gamma-\mathrm{H} 2 \mathrm{AX}$ expression, P21 maybe involved in different cell cycle checkpoints in spermatocytes such as a pre-replication, XY body inactivation, a midpachytene and a metaphase I checkpoints. Finally, in comparison to control, the p21 deficient mouse testis show elevated numbers of apoptosis of elongated spermatids within stages VIII-IX but any difference in the number of spermatogonia mitosis. These results suggest that P21 also may take part in the regulation of the differentiation of the male germ cells and may have a role in spermatogonia not related with a mitotic checkpoint.
\end{abstract}

\section{Keywords}

P21 CDKN1A, Spermatogenesis

${ }^{1}$ Corresponding author: anapedrolaboratories@gmail.com, A.Pedro@hull.ac.uk 
The cyclin dependent kinase inhibitor P21 CDKN1A has been shown to be upregulated during differentiation and after DNA damage in somatic cells (1). Also, it was found that P21 is a factor which maybe important during the meiotic prophase in spermatocytes and repair mechanisms in these cells, but not in the spermatogonial cell cycle (2). Here we further study the role of P21 in the mouse seminiferous epithelium by analysing its expression in mouse testis and by performing apoptosis and proliferation studies in the mouse deficient for $\mathrm{p} 21$.

We started by analysing the expression of P21 CDKN1A in mouse testes paraffin cuts. P21 is expressed in a variable mode in different cell types (spermatogonia, spermatocytes, spermatids, Sertoli cell) located since the basal till the lumen of the seminiferous tubules (fig.1).

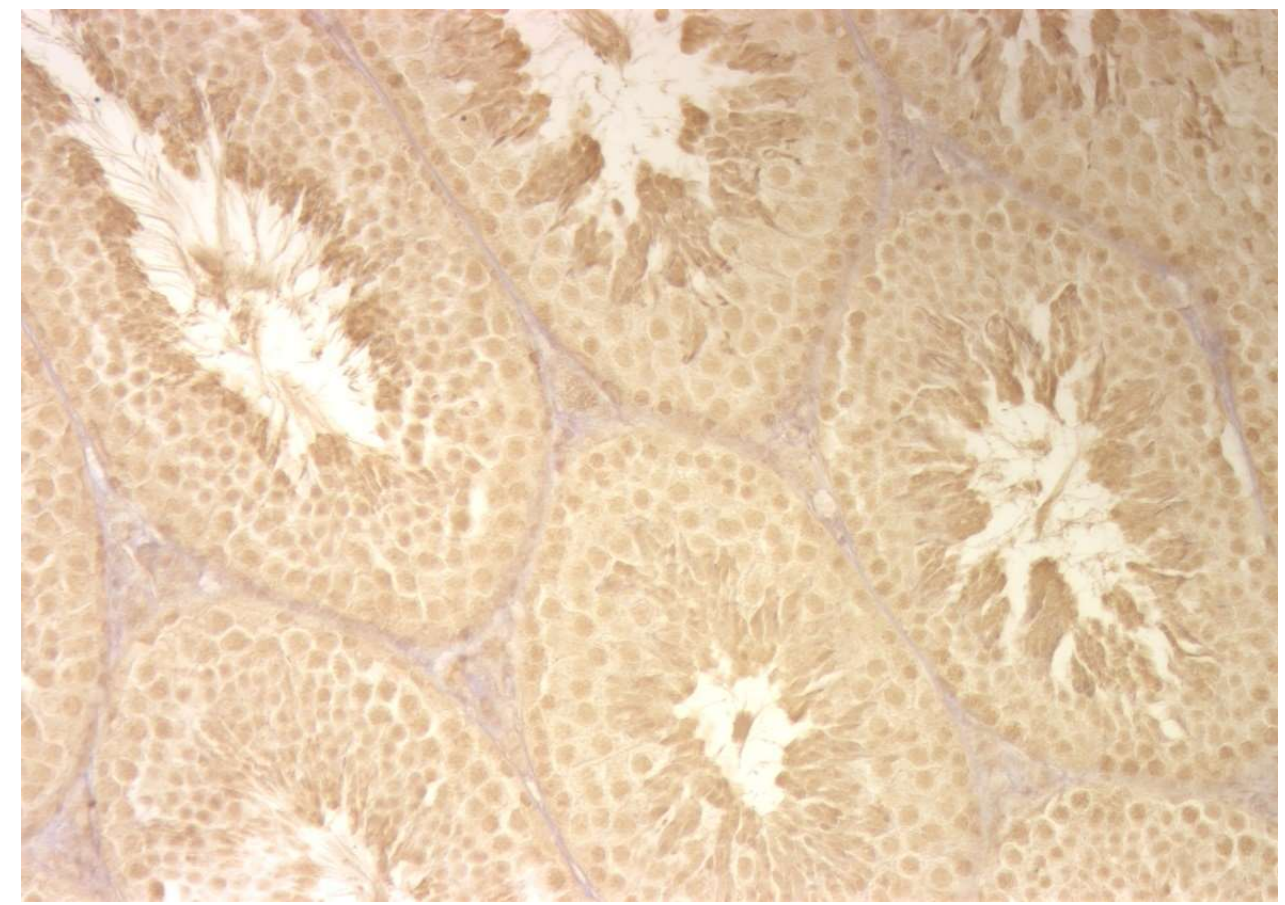

Figure 1: Expression of P21 CDKN1A in the mouse testes (POD, $\mathrm{x} 40$ )

Further, we counted the number of spermatogonia mitotic figures in control and in p21 deficient testis and found there is no significant difference between the number of spermatogonia mitotic figures in control and in $p 21$ deficient testis (9) (not shown).

Next, we analysed de relationships of the expression of P21 CDKN1A with those of $\gamma-\mathrm{H} 2 \mathrm{AX}$ in the mouse meiotic germ cells (fig.2). During preleptotene, when pre-meiotic replication takes place expression of $\mathrm{P} 21$ and $\gamma-\mathrm{H} 2 \mathrm{AX}$ are similar, meaning $\mathrm{P} 21$ maybe halting cell cycle progression while $\gamma$-H2AX identifies sites of stalled replication forks (3). During leptotene, when meiotic doublestrand breaks (DSBs ) occur, $\gamma-\mathrm{H} 2 \mathrm{AX}$ expression is maximal (4) while P21 is much lower, thus allowing cell cycle progression while meiosis is initiated. During zygotene and the zygotenepachytene transition (Z-P), $\quad \gamma-\mathrm{H} 2 \mathrm{AX}$ starts decreasing while P21 raises to a peak of expression at Z-P, when $\gamma-\mathrm{H} 2 \mathrm{AX}$ only appears covering the forming X-Y body (5), suggesting that P21 plays a role in XY body inactivation. Moreover, during pachytene as $\gamma-\mathrm{H} 2 \mathrm{AX}$ continues to fade, $\mathrm{P} 21$ shows another peak of expression where it is supposed it works a mid-pachytene checkpoint monitoring those pachytene spermatocytes with meiotic recombination defects $(6,7) \cdot \gamma$-H2AX totally disappears during diplotene while P21 peaks again at metaphase I (MI), suggesting a role for P21 during a MI checkpoint (8). 


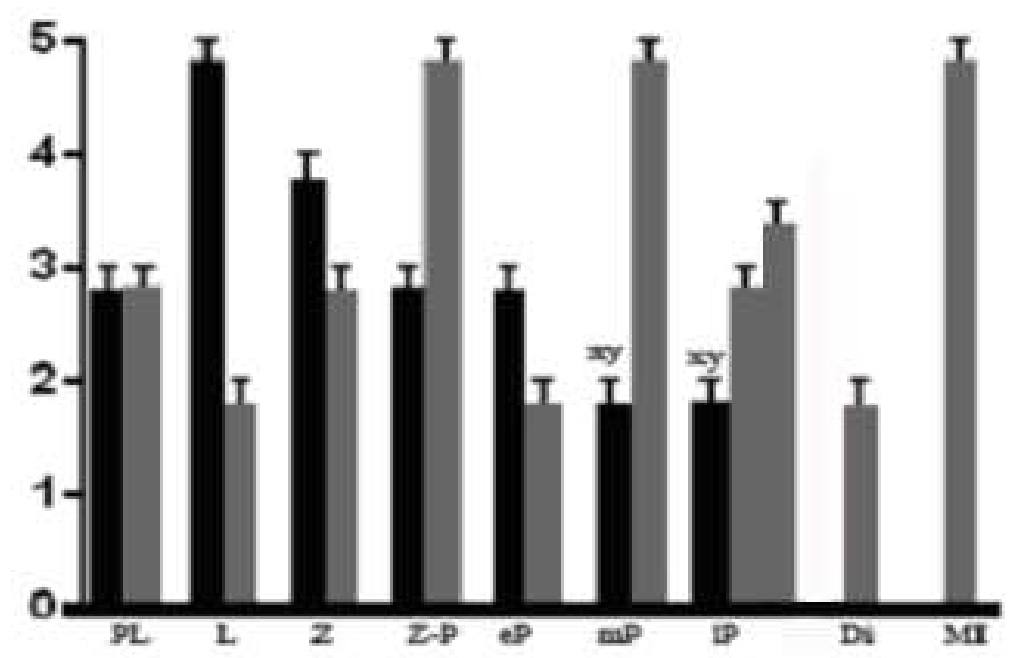

Figure 2: Variation of the expression of P21 (arbitrary units from 0 to 5) in relation with $y-H 2 A X$ in different cell types of mouse testes. Black bars- $\mathrm{Y}-\mathrm{H} 2 \mathrm{AX}$, grey bars - P21. PL - preleptotenes, $\mathrm{L}$-leptotenes, $\mathrm{Z}-$ zygotenes, Z-P - transition zygotene-pachytene, eP - early pachytene, $\mathrm{mP}$ - mid pachytene, IP - late pachytene, $\mathrm{Di}$ - diplotene, $\mathrm{MI}$ - metaphase I. $\mathrm{xy}-\mathrm{y}-\mathrm{H} 2 \mathrm{AX}$ is only present covering the $\mathrm{XY}$ body.

Finally, we quantified apoptosis in the mouse seminiferous epithelium (fig.3). We got to the conclusion that in the p21 deficient mouse there are more apoptosis of elongated spermatids at stages VIII and IX.

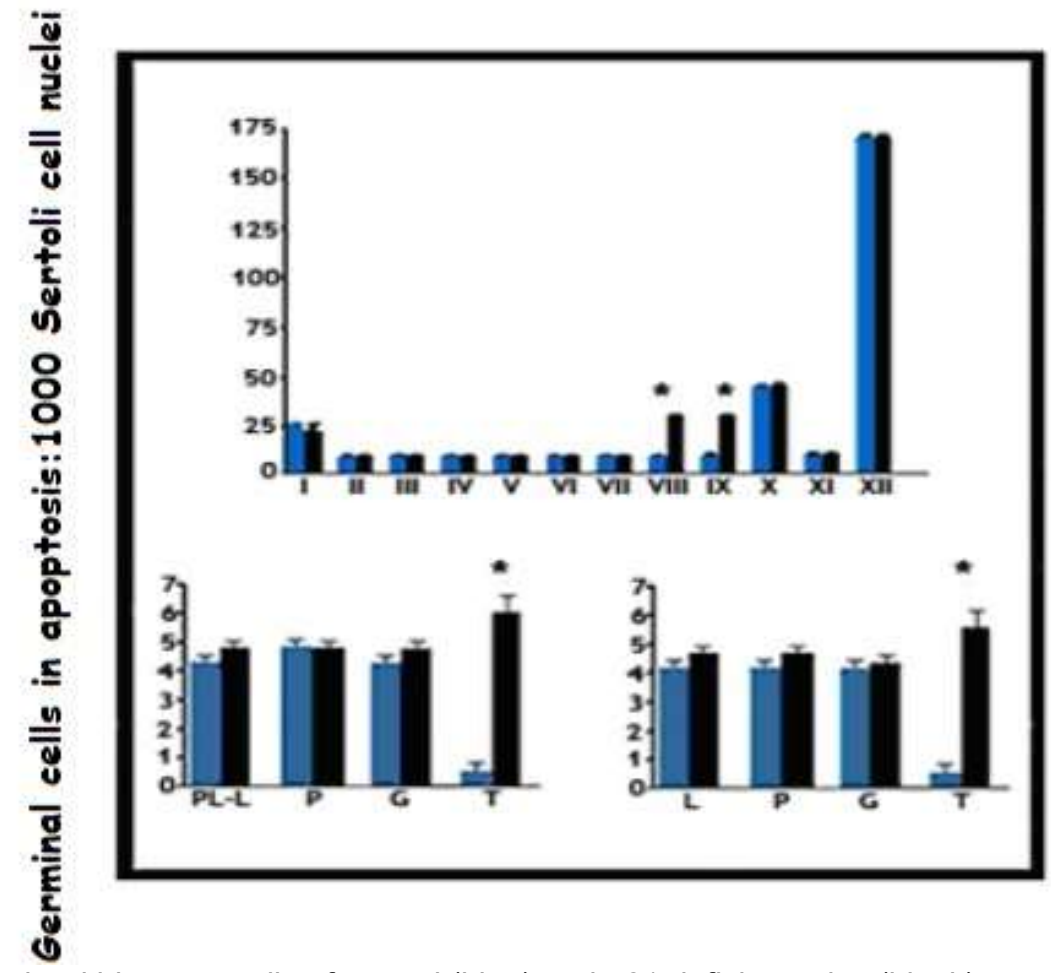

Figure 3: Apoptosis within germ cells of control (blue) and p21 deficient mice (black) per stage and cell types (germ cells in apoptosis per 1000 Sertoli cell nuclei). *, means significant statistical difference, $p<0,05$. The lower diagrams represent apoptosis at stages VIII (PL-L -preleptotene, P- pachytenes, G, spematogonias, T, elongated spermatids) and IX (L-leptotene, P- pachytenes, G, spematogonias, T, elongated spermatids) 


\section{Conclusions}

These results suggest that P21 may take part in the regulation of the differentiation of the male germ cells and may have a role in spermatogonia not related with a mitotic checkpoint. Also, P21 maybe involved in different cell cycle checkpoints in spermatocytes such as a pre-replication, XY body inactivation, a mid-pachytene and a metaphase I checkpoints.

\section{Materials and methods}

This study was performed within the scope of Ana Pedro's PhD Thesis approved on the 07/07/2009 by the Dean of University of Valladolid, Spain.

Mice

We used control and p21 deficient (9) mice with the same background (129sv/C57BL6; 50:50) kindly donated by Manuel Serrano, CNIO, Madrid. The mice testes were dessicated and included in paraffin blocks.

\section{P21 CDKN1A and $\gamma-H 2 A X$ staining}

We used for brightfield P21 study the following antibodies: P21 CDKN1A Neomarkers, ab-9, \#RB032-P0, 1:150), Santa Cruz Biotechnology, Inc; Rabbit ABC Staining System; \#sc-2018. For double immunofluorescence we used mouse anti- $\gamma-\mathrm{H} 2 \mathrm{AX}, 1: 2000$ (Upstate, \#05-636), P21 CDKN1A Neomarkers, ab-9, \#RB-032-P0, 1:150), Santa Cruz Biotechnology, Inc., anti-rabbit Alexa Fluor ${ }^{\circledR} 488$, 1:500 (Molecular Probes, \# A-11034), anti-mouse Alexa Fluor ${ }^{\circledR}$ 488, 1:1500 (Molecular Probes, \# A-21202), anti-rabbit Alexa Fluor ${ }^{\circledR}$ 594, 1:1000 (Molecular Probes \# A-21207), antimouse Alexa Fluor ® 594 (Molecular Probes, \# A-21203)

Apoptosis labelling

For apoptosis labelling, we used the Roche, In situ Cell Death Detection Kit, POD, \# 1684 817).

Statistics

We used Prism program. For apoptosis quantification we analysed 10 paraffin cuts from p21 deficient mouse and 10 paraffin cuts from control mouse, counter-stained with PAS and count the number of apoptosis in 30 tubules of each stage in agreement with Oakberg (1956). For mitosis, we counted the number of mitotic figures of spermatogonia in 5 non-consecutive tubules of the stages $I$, $I V, V I, I X, X y$ XII both in control and p21 deficient mouse. We calculated the percentageof mitosis in relation to the average number of Sertoli cell in control and in p21 deficient mouse.

\section{References}

1. Kreis et al. The Multifaceted p21 (Cip1/Waf1/CDKN1A) in Cell Differentiation, Migration and Cancer Therapy. Cancers (Basel). 2019 Aug 21;11(9):1220.

2.Beumer et al. 1997. P21(Cip1/WAF1) expression in the mouse testis before and after $X$ irradiation. Mol Reprod Dev. 1997 Jul;47(3):240-7.

3. Gagou et al. Enhanced H2AX Phosphorylation, DNA Replication Fork Arrest, and Cell Death in the Absence of Chk1. Mol Biol Cell. 2010 Mar 1; 21(5): 739-752.

4. Hunter et al. H2AX illuminates meiosis. Nature Genetics 27,236-238(2001)

5. Abe et al. 2020. The Initiation of Meiotic Sex Chromosome Inactivation Sequesters DNA Damage Signaling from Autosomes in Mouse Spermatogenesis. Current Biology.Volume 30, Issue 3,3 February 2020, Pages 408-420.e5

6.Ashley et al., 2004, The mammalian mid-pachytene checkpoint: meiotic arrest in spermatocytes with a mutation in Atm alone or in combination with a Trp53 (p53) or Cdkn1a (p21/cip1) mutation. Cytogenet Genome Res. 2004;107(3-4):256-62.

7. Crichton et al.2018 Defects in meiotic recombination delay progression through pachytene in Tex19.1-/-mouse spermatocytes. Chromosoma 127,437-459

8. Eaker et al. 2001. Evidence for meiotic spindle checkpoint from analysis of spermatocytes from Robertsonian-chromosome heterozygous mice. J Cell Sci (2001) 114 (16): 2953-2965.

9. Brugarolas et al. 1995. Radiation-induced cell cycle arrest compromised by p21 deficiency. Nature377,552-557(1995) 